\title{
Up-dates on Cardio-Circulatory Support
}

\author{
ATTAULLAH KHAN NIAZI ${ }^{1}$, JULIA STEHLI ${ }^{2}$, MUHAMMAD MUNEEB $^{3}$, AMMAR HAMEED KHAN $^{4}$, AHSAN ARIF $^{5}$, KANZA SANA UMER $^{6}$, \\ SANA RASHEED ${ }^{7}$ \\ ${ }^{1}$ Assistant Professor, Department of Cardiovascular Surgery, Shalamar Medical \& Dental College, Lahore. \\ ${ }^{2}$ Department of Interventional Cardiology, University hospital Charlottesville, VA, USA \\ ${ }^{3}$ Assistant Professor, Department of Cardiology, Shalamar Medical \& Dental College, Lahore \\ ${ }^{4}$ Professor of Cardiovascular Surgery, Sialkot Medical College, Sialkot \\ ${ }^{5}$ Consultant Cardiac Surgeon \\ ${ }^{6}$ Clinical Psychologist, Kingdom of Saudi Arabia \\ ${ }^{7}$ Consultant Gynaecologist, Jinnah Hospital, Lahore \\ Correspondence to Dr. Attaullah Khan Niazi, Email: drattaniazi@yahoo.com
}

\section{INTRODUCTION}

In-hospital mortality rates have approved in the recent past ${ }^{1} 6$ to 12 -month death persists at approximately $50 \%^{2}$. The goal of treatment is to reestablish tissue perfusion while closely monitoring hemodynamics. Provisional automatic cardiovascular support is a mainstay of the management of patients who cannot be stabilized with medical therapy.

Apart from the acute setting, automatic cardiovascular support is also used in the mid- and long-term treatment of heart failure patients. This can either be until heart transplant or life-long in patents who are not candidates for heart transplant. Further it is an essential tool of open-heart surgery. In the present review we will give an overview and update of the different devices for mechanical cardiac support.

Non-mechanical Cardio-Circulatory Support includes intravenous fluids and pharmacological treatment with vasopressors and inotropic agents. Norepinephrine is mainly a vasopressor, but also has other activities such as inotropy. It serves as first line agent, particularly in the acute setting of a cardiogenic shock. A recent randomized trial comparing Norepinephrine versus Epinephrine in cardiogenic shock after myocardial infarction found that Norepinephrine appeared to be a safer choice than Epinephrine ${ }^{3}$. Vasopressin causes less pulmonary vasoconstriction than norepinephrine and is therefore might better as a first line vasopressor compared to Norepinephrine ${ }^{4}$. Dobutamine, a beta agonist is frequently being used for cardiogenic shock, acting as inotropic agent. Levosimendan is a Calcium sensitizer and often used in patients with acute decompensation of chronic heart failure.

Mechanical Cardio-Circulatory Support: Ventricular assist devices (VAD) can be categorized according to period of provision, according to the mechanism of the device or according to which ventricle is being supported. The duration of support is either bridge- to-device, bridge-to-transplant, bridge-to-recovery or, the latter for short-term assist devices if an upgrade of a long-term device is planned. If the device is planned for permanent use, it is called 'destination therapy'. Again, these time spans can be summarized as short-, intermediate- or long-term support devices.

Most patients $(85 \%)$ receive an assist device for the left ventricle (LVAD), with a underground receiving biventricular (biVAD) provision or a total artificial heart and even less having only the right ventricle supported (RVAD) ${ }^{5}$

The following summary gives an overview according to the mechanism of the device. and Intra-aortic balloon pump will be described first, since these two do not classify as ventricular assist device, compared to the other mechanical cardio-circulatory support systems.

Short- and intermediate cardio circulatory support devices Extracorporeal membrane oxygenation (ECMO): One of the most widely used circulatory support systems is the extracorporeal membrane oxygenation device which is used by interventional Cardiologists, Cardio-thoracic surgeons and intensive care specialists alike.

Function and Usage: Venous-blood vessel (V-A) V-An ECMO is fundamentally utilized as a treatment for intense serious heart as well as respiratory disappointment. The utilization isn't limited to grown-up respiratory and cardiovascular infection, yet additionally incorporates youngsters with heart sickness (Table 1, adjusted from Butt et $\left.\mathrm{al}^{6}\right)$. V-An ECMO can work with the protected utilization of different medicines, like heart medical procedure or interventional cardiology methodology. The utilization of V-An ECMO in grown-up cardiorespiratory disappointment as an extension to long haul support with ventricular help gadgets and relocate programs is likewise possible ${ }^{7}$. Extra-bodily cardiopulmonary revival is one more original utilization of $\mathrm{V}$-An ECMO. In patients with heart failure, V-An ECMO can give more noteworthy blood stream and oxygen conveyance during than chest compressions. In addition, V-An ECMO settles shaky patients to such an extent that treatments considered hazardous, for example, hemodialysis in babies or backing of imperative organ work during rewarming from incidental hypothermia can be embraced safely ${ }^{8,9}$. V-V ECMO can be utilized as a drawn out extension to work with lung transplantation ${ }^{10}$. Lastly, V-An ECMO can be coordinated into various organ support treatments i.e., during a septic shock.

Table 1: Adjusted from Butt et al
\begin{tabular}{|l|c|c|c|}
\hline & $\mathbf{n}$ & $\begin{array}{c}\text { Survived ECMO } \\
\text { support }\end{array}$ & $\begin{array}{c}\text { Survived to hospital } \\
\text { discharge }\end{array}$ \\
\hline Neonatal & \multicolumn{3}{|c|}{} \\
\hline Respiratory & $28^{\prime} 723$ & $84 \%$ & $74 \%$ \\
\hline Cardiac & $6^{\prime} 269$ & $62 \%$ & $41 \%$ \\
\hline ECPR & $1^{\prime} 254$ & $64 \%$ & $41 \%$ \\
\hline Paedriatic & \multicolumn{5}{|l|}{} \\
\hline Respiratory & $7^{\prime} 210$ & $66 \%$ & $58 \%$ \\
\hline Cardiac & $8^{\prime} 021$ & $67 \%$ & $51 \%$ \\
\hline ECPR & $2 ' 788$ & $55 \%$ & $41 \%$ \\
\hline Adults & $9^{\prime} 102$ & $66 \%$ & $58 \%$ \\
\hline Respiratory & $7^{\prime} 850$ & $56 \%$ & $41 \%$ \\
\hline Cardiac & $2 ' 379$ & $40 \%$ & $30 \%$ \\
\hline ECPR &
\end{tabular}

Current data on outcomes: Endurance among patients with cardiogenic shock on V-An ECMO support stays unassuming, with generally speaking emergency clinic death paces of $55 \%^{11}$. Patients with possibly reversible reasons for shock, like fulminant myocarditis or essential join disappointment, have preferred endurance over patients with cardiogenic shock after a medical procedure or intense myocardial infarction ${ }^{12}$. Patients for whom VAn ECMO is conveyed during or following heart failure have a particularly helpless guess with a mortality underneath $30 \%{ }^{11}$. PreECMO hazard factors autonomously connected with helpless results incorporate more established age, female sex, and higher weight list, just as markers of disease seriousness including renal, hepatic, or focal sensory system brokenness, longer term of mechanical ventilation, raised lactate levels, and diminished prothrombin activity ${ }^{13}$. The appropriate selection of patients for V-A ECMO is a challenge considering that assessment of a potential reversibility of the shock state can be difficult.

Most common complications are leg ischemia, bleeding and infection. The rate of complications is with $30-40 \%$ rather high, which limits the long-term use of V-A ECMO. All things considered, further such advances will decrease entanglement rates, work with more far reaching reception of the innovation in center and big league salary nations, and further develop results from stubborn heart, lung, and multi-organ failure ${ }^{14}$. 
Updates: Applications: The main issues over the most recent couple of years for clinicians engaged with ECMO rely upon the kind and experience of the ECMO program and the emergency clinic in which ECMO is performed. New projects are zeroing in on standard employments of ECMO, frameworks for safe use and sending of ECMO, the board of patients on ECMO and getting signs and contraindications. Long-standing projects, then again, are zeroing in on further developing results by considering elective ECMO methodologies like ordinary or high stream for septic shock, different kinds of fringe or trans-thoracic cannulation, and prior commencement of ECMO. This additionally incorporates therapy of different patient gatherings like patients with insusceptible concealment or disease, pregnant ladies, or the elderly ${ }^{15}$. Complex chemotherapy or immunotherapy regimens can cause a serious foundational fiery reaction requiring cardiovascular support ${ }^{16}$.

Timing: The execution of V-An ECMO is changing as the innovation keeps on improving. Maybe than being utilized if all else fails or salvage treatment, it has turned into a standard treatment and is progressively being executed before throughout the illness trying to limit multi-organ disappointment. In out-of-hospital arrests, attempts to shorten time to restoration of circulation have been made with 'in the field' V-A ECMO cannulation, such as in France. The first randomised prospective trial comparing V-A ECMO implantation 'in-field' versus in- hospital has recruited 201 patients in France (APACAR2 trial - A Comparative Study between a prehospital and an In- hospital Circulatory Support Strategy [ECMO] in Refractory Cardiac Arrest, NCT02527031, ClinicalTrials.gov). Enrolment has been completed, however, results are still outstanding. Of course, extra-corporeal cardiopulmonary resuscitation is also performed during $\mathrm{PCl}$ in the cardiac catheterization laboratories and in the ICU.

Device: Fresher cannulas with further developed stream qualities or more biocompatible plastics are being fostered that will additionally work on the wellbeing of ECMO. A definitive objective is to have a circuit that is completely biocompatible with no requirement for anticoagulation and no danger of thromboembolism or drain. Self-controlled stream request circles are ideally just age away ${ }^{12}$.

Covid-19: ECMO in acute respiratory distress syndrome ${ }^{18}$ is currently used as therapy for Covid-19 patients if prone ventilation and other supportive therapies have failed. The frequency of this rescue strategy has varied among centres with rates from $1 \%$ to $24 \%{ }^{19,20,21}$

Intra-aortic balloon pump (IABP) ${ }^{22}$ : This is mainly because of its easy use, high safety profile and lower cost compared to other more sophisticated devices.

\section{Current data on outcome:}

Updates: A relatively new field of interest is the use of IABP in patients receiving $E C M O$. The combination makes sense from a physiological standpoint: IABP can help to reduce the after-load which is often increased by the ECMO. However, the data about is remains controversial ${ }^{25}$.

Impella:

Impella (AbioMed, Danvers, MA, USA) is the new kid on the block of cardiac support devices that can be used by interventional cardiologist. Indications for Impella include cardiovascular support during high risk interventions (mostly $\mathrm{PCl}$ ) or unloading of the left ventricular in the setting of ST elevation myocardial infarction (STEMI).

Current data on outcomes: Similarly, the Impella device did not show any significant benefits in the PROTECT II trial, which randomized patients with triple vessel disease or unprotected left main undergoing $\mathrm{PCl}$ to Impella versus $\mathrm{IABP}^{29}$. However, real world experience shows that multiple high risk $\mathrm{PCl}$ cases would not be performed if it was not for Impella support. More studies are needed to support this theory ${ }^{28}$.

Updates: Recently the concept that unloading of the ventricle with a mechanical support device in the setting of STEMI could reduce infarct size and prevent reperfusion injury gained interest. In prospective trial patients with STEMI were randomized to either direct $\mathrm{PCl}$ or a 30-minute delay to revascularization during which the left ventricle was unloaded with an Impella CP device. The study showed that unloading with the Impella device during a 30minute period was safe, paving the way for larger randomized trials testing this concept ${ }^{30}$.

\section{Right ventricular assist devices (RVADs)}

Function and Usage: Intense right ventricular disappointment might happen in various settings, including intense myocardial dead tissue, myocarditis, intense biventricular decompensated cardiovascular breakdown, intense aspiratory embolism, pneumonic hypertension, post-cardiotomy and posttransplantation ${ }^{33}$. However, the majority of patients needing an RVAD are patients have been treated for left heart failure. Almost $50 \%$ of patients show right heart failure after LVAD implantation requiring right ventricular support, sometimes within the first two weeks post-operation ${ }^{34}$. Interestingly, the right ventricle often recovers within weeks. Consequentially, the majority of devices are made for temporary use. Currently both surgical and percutaneous options are available.

Such options include, but are not limited to, the HeartWare "Medtronic, Dublin, Ireland) and Jarvik 2000 (Jarvik Heart, New York, NY, USA" devices. The disadvantage of surgical RVADs is also that often repeat sternotomy is necessary if the RVAD is not implanted at the same time as the LVAD. Further, because most of them are only for short- or intermediate-term support, a second surgery to remove the device is necessary once the patient can be weaned. On the contrary, the Impella RP device is implanted percutaneously with a flow rate of up to $5.0 \mathrm{l} / \mathrm{min}$ for a support of up to 2 weeks.

Intermediate and long-term cardio circulatory support devices Left- and biventricular assist devices (VADs)

Function and Usage: With an LVAD, Early VADs as the "HeartMate XVE (Thoratec, Pleasanton, CA, USA)" imitating the pulsatile flow of the heart. They were mainly used for patients awaiting cardiac transplantation. Second and third generation devices abandoned the concept of pulsatile flow but instead adapted a continuous axial flow through a valve-less pump with a variable magnetic field. These novel qualities provide longer durability, better hemodynamics, less thrombosis risk, less noise, smaller device size and possibly lower driveling infection risk. Models include Jarvik 2000, CentriMag (Abbott, Chicago, IL, USA), Incor (Berlin Heart, Berlin, Germany). Notably, most popular models are HeartMate 3 (Thoratec) and Heart Ware. The HeartMate 3 has a magnetically levitated core system called Full MagLev technology, which reduces haemolysis \& thrombosis risk.

The overall use of LVAD has increased substantially in recent years, including patients receiving a VAD as bridge-totransplant. $20 \%$ of them are support by an LVAD because of stagnating heart donors among other reasons ${ }^{38}$

Current data on outcomes: Despite the advantages of later generation VADs, complications such as driveline infection, thrombosis, bleeding and haemolysis still persist in all devices ${ }^{39}$.

A randomized trial comparing Heart Mate $I I$ and HeartWare demonstrated similar rates in the main endpoint which was survival [40] Patients with the HeartWare had higher rates of stroke $(29 \%$ versus $12 \%$ ), right heart failure, and sepsis, but less device malfunction or device failure compared with patients in the HeartMate II arm.

Applications: VADs were initially not promoted as bridge-torecovery therapy. However, more recently, the concept of left ventricular recovery during LVAD therapy has become more popular. Studies could demonstrate that mechanical unloading with an LVAD while applying specific pharmacological therapy could lead to recovery of the heart and eventually removal of the assist device ${ }^{41,42}$.

New developments: Due to the still high complication rates, research for improvements in VADs is ongoing. Alternative powering with the aim to eliminate extracorporeal power requirements is one of the main goals. This would not only reduce the risk of driveline infections, but also lead to better quality of life of patients with totally implantable devices. Transcutaneous energy transmission technology and electrically stimulated skeletal muscle 
as endogen power source are currently under investigation, however, clinically not available yet. In an attempt to reduce both bleeding as a result of anticoagulant therapy and thrombosis, devices with zero blood contact have been designed, however, they are as well still in the development phase.

\section{CONCLUSIONS}

Acute and chronic heart failure are diseases with persistent highmortality, clinically presents as multiorgan failure scenario, cardiogenic shock and death. Advances in support systems always assistance in better outcomes, however, the struggle with complications is persistent and real. Patient consequence is also center precise and very reliant on native issues. The cardiac community should remain to part outcomes and information in an effort to recover patient results. Last but not least, Selection of appropriate device is very crucial and multiple factors should be consider, including the stage of cardiogenic shock, Target management, High risk patient and technical limitations.

\section{REFERENCES}

1. Diepen, S.v., et al., Contemporary Management of Cardiogenic Shock: A Scientific Statement from the American Heart Association. Circulation, 2017. 136(16): p. e232-e268.

2. Ouweneel, D.M., et al., Percutaneous Mechanical Circulatory Support Versus Intra-Aortic Balloon Pump in Cardiogenic Shock After

3. Acute Myocardial Infarction. J Am Coll Cardiol, 2017. 69(3): p. 278

4. Levy, B., et al., Epinephrine Versus Norepinephrine for Cardiogenic Shock After Acute Myocardial Infarction. J Am Coll Cardiol, 2018. 72(2):p.173-182.

5. Gordon, A.C., et al., The cardiopulmonary effects of vasopressin compared with norepinephrine in septic shock. Chest, 2012. 142(3): $p$. 593-605.

6. Cleveland, J.C., Jr., et al., Survival after biventricular assist device implantation: an analysis of the Interagency Registry for Mechanically Assisted Circulatory Support database. J Heart Lung Transplant, 2011. 30(8): p. 862-9.

7. Butt, W. and G. MacLaren, Extracorporeal membrane oxygenation 2016: an update. F1000Res, 2016. 5.

8. Abrams, D., A. Combes, and D. Brodie, Extracorporeal membrane oxygenation in cardiopulmonary disease in adults. J Am Coll Cardiol, 2014. 63(25 Pt A): p. 2769-78.

9. Shebani, S.O., et al., Radiofrequency ablation on veno-arterial extracorporeal life support in treatment of very sick infants with incessant tachymyopathy. Europace, 2015. 17(4): p. 622-7.

10. Sawamoto, K., et al., Outcome from severe accidental hypothermia with cardiac arrest resuscitated with extracorporeal cardiopulmonary resuscitation. Am J Emerg Med, 2014. 32(4): p. 320-4

11. Inci, I., et al., Outcome of Extracorporeal Membrane Oxygenation as a Bridge To Lung Transplantation: An Institutional Experience and Literature Review. Transplantation, 2015. 99(8): p. 1667-71.

12. (ELSO), E.-c.L.S.O.; Available from: https://www.elso.org/Registry/ Statistics/InternationalSummary.aspx

13. Keebler, M.E., et al., Venoarterial Extracorporeal Membrane Oxygenation in Cardiogenic Shock. JACC Heart Fail, 2018. 6(6): p. 503-516.

14. Marasco, S.F., et al., Review of ECMO (extra corporeal membrane oxygenation) support in critically ill adult patients. Heart Lung Circ, 2008. 17 Suppl 4: p. S41-7.

15. Trzeciak, S., et al., Early increases in microcirculatory perfusion during protocol-directed resuscitation are associated with reduced multi-organ failure at $24 \mathrm{~h}$ in patients with sepsis. Intensive Care Med, 2008. 34(12): p. 2210-7.

16. Sharma, N.S., et al., Modern use of extracorporeal life support in pregnancy and postpartum. Asaio j, 2015. 61(1): p. 110-4.

17. Worku, B., et al., Extracorporeal Membrane Oxygenation as a Bridge through Chemotherapy in B-Cell Lymphoma. J Extra Corpor Technol, 2015. 47(1): p. 52-4

18. Maxhera, B., et al., Survival predictors in ventricular assist device patients with prior extracorporeal life support: selecting appropriate candidates. Artif Organs, 2014. 38(9): p. 727-32.

19. Combes, A., et al., Extracorporeal Membrane Oxygenation for Severe Acute Respiratory Distress Syndrome. N Engl J Med, 2018. 378(21): p. $1965-1975$

20. Yang, X., et al., Clinical course and outcomes of critically ill patients with SARS-CoV-2 pneumonia in Wuhan, China: a single-centered, retrospective, observational study. Lancet Respir Med, 2020.

21. Grasselli, G., et al., Baseline Characteristics and Outcomes of 1591 Patients Infected With SARS-CoV-2 Admitted to ICUs of the Lombardy Region, Italy. Jama, 2020.

22. https://www. elso.org/Registry/FullCOVID19RegistryDashboard aspx.

23. Ponikowski, P., et al., 2016 ESC Guidelines for the diagnosis and treatment of acute and chronic heart failure: The Task Force for the diagnosis and treatment of acute and chronic heart failure of the European Society of Cardiology (ESC). Developed with the special contribution of the Heart Failure Association (HFA) of the ESC. Eur J Heart Fail, 2016. 18(8): p.891-975.

24. Thiele, $\mathrm{H}$., et al., Intraaortic balloon support for myocardial infarction with cardiogenic shock. N Engl J Med, 2012. 367(14): p. 1287-96

25. Romeo, F., et al., The outcome of intra-aortic balloon pump support in acute myocardial infarction complicated by cardiogenic shock according to the type of revascularization: a comprehensive metaanalysis. Am Heart J, 2013. 165(5): p. 679-92.

26. Wang, D., et al., Does concurrent use of intra-aortic balloon pumps improve survival in patients with cardiogenic shock requiring venoarterial extracorporeal membrane oxygenation? Interact Cardiovasc Thorac Surg, 2020. 30(2): p. 312-315.

27. Mandawat, A. and S.V. Rao, Percutaneous Mechanical Circulatory Support Devices in Cardiogenic Shock. Circ Cardiovasc Interv, 2017. 10(5).

28. Seyfarth, M., et al., A randomized clinical trial to evaluate the safety and efficacy of a percutaneous left ventricular assist device versus intra-aortic balloon pumping for treatment of cardiogenic shock caused by myocardial infarction. J Am Coll Cardiol, 2008. 52(19): p. 1584-8.

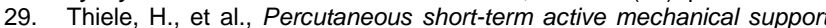
devices in cardiogenic shock: a systematic review and collaborative meta- analysis of randomized trials. Eur Heart J, 2017. 38(47): p. 3523.

30. O'Neill, W.W., et al., A prospective, randomized clinical trial of hemodynamic support with Impella 2.5 versus intra-aortic balloon pump in patients undergoing high-risk percutaneous coronary intervention: the PROTECT II study. Circulation, 2012. 126(14): p. 1717-27.

31. Kapur, N.K., et al., Unloading the Left Ventricle Before Reperfusion in Patients With Anterior ST-Segment-Elevation Myocardial Infarction. Circulation, 2019. 139(3): p. 337-346.

32. Thiele, H., et al., Randomized comparison of intra-aortic balloon support with a percutaneous left ventricular assist device in patients with revascularized acute myocardial infarction complicated by cardiogenic shock. Eur Heart J, 2005. 26(13): p. 1276-83.

33. Kar, B., et al., The percutaneous ventricular assist device in severe refractory cardiogenic shock. J Am Coll Cardiol, 2011. 57(6): p.688-96.

34. Saeed, D., Right Ventricular Failure and Biventricular Support Strategies.

35. Cardiol Clin, 2018. 36(4): p. 599-607

36. Kiernan, M.S., et al., Early Right Ventricular Assist Device Use in Patients Undergoing Continuous-Flow Left Ventricular Assist Device Implantation: Incidence and Risk Factors From the Interagency Registry for Mechanically Assisted Circulatory Support. Circ Heart Fail, 2017. 10(10)

37. Sultan, I., A. Kilic, and A. Kilic, Short-Term Circulatory and Right Ventricle Support in Cardiogenic Shock: Extracorporeal Membrane Oxygenation, Tandem Heart, CentriMag, and Impella. Heart Fail Clin, 2018. 14(4): p. 579-583.

38. Anderson, M.B., et al., Benefits of a novel percutaneous ventricular assist device for right heart failure: The prospective RECOVER RIGHT study of the Impella RP device. J Heart Lung Transplant, 2015. 34(12): p. 1549-60.

39. Kapur, N.K., et al., Mechanical Circulatory Support Devices for Acute Right Ventricular Failure. Circulation, 2017. 136(3): p. 314-326.

40. Aurora, P.,et al., The Registry of the International Society for Heart and Lung Transplantation: thirteenth official pediatric lung and heart-lung transplantation report--2010. J Heart Lung Transplant, 2010. 29(10): p. 1129-41.

41. Lima, B., et al., Controversies and Challenges of Ventricular Assist Device Therapy. Am J Cardiol, 2018. 121(10): p. 1219-1224.

42. Rogers, J.G., et al., Intrapericardial Left Ventricular Assist Device for Advanced Heart Failure. N Engl J Med, 2017. 376(5): p. 451-460.

43. Birks, E.J., et al., Left ventricular assist device and drug therapy for the reversal of heart failure. N Engl J Med, 2006. 355(18): p. 1873-84.

44. Birks, E.J., et al., Reversal of severe heart failure with a continuousflow left ventricular assist device and pharmacological therapy: a prospective study. Circulation, 2011. 123(4): p. 381-90. 